\section{Transfer RNA}

\section{J.P. Goddard}

Transfer RNA: Structure, Properties, and Recognition. Edited by P.R. Schimmel, D. Söll, and J.N. Abelson. Pp.577. (Cold Spring Harbor Laboratory: New York, 1979.) $\$ 60$.

NEARLY a quarter of a century has passed since the existence of the small adapter molecules known as transfer RNA was predicted. Their subsequent study has been motivated by their properties. They are relatively small and their central role in protein synthesis involves many specific interactions with proteins and nucleic acids. Furthermore they have since been strongly implicated in regulation of gene expression. The extent of this study is reflected in the fact that two volumes - the second is on biological aspects - were found necessary to fulfil the aim of summarising the knowledge of tRNA up to about the end of 1978 .

The means to that end is, as in previous Cold Spring Harbor monographs, a series of articles by specialists in a particular aspect of tRNA who participated in the Transfer RNA Meeting at Cold Spring Harbor in August 1978. These articles have been supplemented by four appendices on tRNA sequences and a proposed numbering scheme, on modified nucleosides and their chromatographic mobilities, and the characteristics of aminoacyl-tRNA synthetases. This first volume contains some thirty-one articles covering aspects of primary structure and isolation, crystal structure analysis, solution studies, aminoacyl-tRNA synthetases, synthetase recognition, and interaction with the ribosome.

Unlike several of its predecessors, this monograph contains no general review. My immediate reaction to this omission was to praise the economy of the editors in not adding to the several reviews published in the last couple of years. However, a nonspecialist seeking in this volume a thumbnail sketch to tRNA research will be disappointed. A short article expanding the contents of the brief preface by putting each article in its context and perhaps citing other recent reviews would have added less than one per cent to the volume's bulk and price but would have increased its value both to new workers in the field and to scientists from other specialities.

This lack is compensated to a large extent by articles which have extensive bibliographies. This is particularly true of the articles beginning each section which are written by the session chairmen at the meeting who refer to articles later in their section. The invited contributors of articles have usually endeavoured to present not only their own work but also that of other workers in the field. As the writers are, in the main, reporting topics on which they are among the leading experts, many quite reasonably present reviews of their past few years' work interspersed with that of others. This occasionally leads to a onesided interpretation in areas where there is currently disagreement, e.g. the structure of the $E$. coli ribosome. However, the volume is generally notable for the generosity and objectiveness of the writers in reporting views and ideas contrary to their own. These virtues are not purchased at the cost of dull uniformity. Each article retains its individuality and yet the volume forms a coherent unit.

In the absence of a moratorium, it is fortunately not possible to produce "the

definitive reference work on transfer RNA". Even the proposed numbering system of nucleosides in tRNAs (Appendix IA), so eminently reasonable a year ago, looks strangely dated in the light of the structure of the tRNAs in our own mitochondria. However, this volume is the best we can hope for - a summary of the major results of past tRNA research with hints of future solutions to outstanding problems. It should prove stimulating reading to all those working in tRNA research, and those working in other aspects of gene expression will find many articles of value.

J. P. Goddard is a Lecturer in Biochemistry at the University of Glasgow, UK.

\section{Unifying the forces of nature}

\section{Malcolm MacCallum}

Elie Cartan and Albert Einstein: Letters on Absolute Parallelism, 1929-32. Edited by R. Debever. Translated by J. Ritter and J. Leroy. Pp. 233. (Princeton University Press: Princeton, New Jersey, and Guildford, UK, 1979.) £11.40.

I FOUND these 38 letters (and one postcard) an enjoyable and interesting read, but it is difficult to discern the intended readership, and so I do not know to whom I could recommend the book. The reader needs to be polyglot. Einstein wrote in German, Cartan in French. Both are translated, Ritter presumably translating Einstein's letters and Leroy those of Cartan. The preface and notes by the editor, the wellknown Belgian relativist, Robert Debever, are, however, given only in French. The translations are passable, though Leroy's seems to show that English is not his mother tongue. The reader also needs a good knowledge of the background, and the energy to read the references, as the book is not self-contained. The editor even omits one manuscript note because a slightly modified version was published, and he gives only bibliographical details of most of the printed material the correspondents exchanged.

The letters cover three main topics. The first is the writing of a historical note on spaces with absolute parallelism which Cartan drafted in only two weeks in May 1929. It appeared, accompanying Einstein's paper on his attempt at a unified field theory using such spaces, in 1930; Einstein had formulated his ideas in ignorance of the mathematical literature. The second, covered by 26 letters between 3 December 1929 and 17 February 1930, deals with alternative systems of field equations in such spaces, and qualitative features of such systems of partial differential equations linear in first derivatives. Cartan showed there was another set of equations with the same mathematical properties as Einstein's (involutary character, determinacy of the Cauchy problem, and arbitrariness of initial conditions), and Einstein gave reasons for thinking them less physically satisfactory. This discussion gradually narrowed to the third subject, the question of whether one could define the 'strength' of a set of such equations by counting freely specifiable functions of $r$ variables for every $r$ up to some maximum. Cartan argued that only the maximal $r$ gave a meaningful result. The two authors never agreed, even in the seven later letters exchanged in 1931 and 1932. By 1932 Einstein had abandoned distant parallelism for the Einstein-Mayer theory, and the only later outcome is the much modified idea of 'strength' used in The Meaning of Relativity (fifth edition, Appendix II) which Debever does not cite.

What use, then, is the book? The modern reseacher, seeking a unified field theory, will find little of direct relevance to present efforts in this direction. The professional historian of science would probably prefer to consult the original manuscripts. The general reader may be put off by the effort required of him. If he is not, then he may be rewarded in several ways. There are interesting personal sidelights on the characters. The speed of the mail, and the authors' energy (especially as Einstein was 50 and Cartan $60)$ must be admired. There is much to learn from Einstein's attitude to physical problems and from Cartan's clear and careful mathematical expositions, and some reassurance for lesser mortals in their occasional mistakes. Principally, one can understand how two great minds worked in developing an (albeit unsuccessful) attempt at one of the greatest problems in physics, the unification of the forces of nature.

Malcolm MacCallum is a Lecturer in Applied Mathematics at Oueen Mary College, University of London, $U K$. 